\title{
Latest results on rare kaon decays from the NA48/2 experiment at CERN
}

\author{
M. Raggi ${ }^{a, b}$ for the NA48/2 collaboration * \\ ${ }^{a}$ Department of Physics Sapienza University, Piazzale Aldo Moro 5, Roma, Italy \\ ${ }^{b}$ INFN sezione di Roma, Piazzale Aldo Moro 5, Roma, Italy \\ E-mail: mauro.raggi@roma1.infn.it
}

The NA48/2 experiment at CERN reports the first observation of the $K^{ \pm} \rightarrow \pi^{ \pm} \pi^{0} e^{+} e^{-}$decay from an exposure of $1.7 \times 10^{11}$ charged kaon decays recorded in 2003-2004. A sample of 4919 candidates with $4.9 \%$ background contamination allows the determination of the branching ratio in the full kinematic region. The study of the kinematic space shows evidence for a structure dependent contribution in agreement with predictions based on chiral perturbation theory. Several $\mathrm{P}$ - and CP-violating asymmetries are also evaluated. The most precise measurement of the charged kaon semi-leptonic form factors obtained by NA48/2 with 4.4 million Ke3 and 2.3 million K $\mu 3$ events collected in 2004 will also be presented.

40th International Conference on High Energy physics - ICHEP2020

July 28 - August 6, 2020

Prague, Czech Republic (virtual meeting)

*G. Anzivino, R. Arcidiacono, W. Baldini, S. Balev, J.R. Batley, M. Behler, S. Bifani, C. Biino, A. Bizzeti, B. BlochDevaux, G. Bocquet, N. Cabibbo, M. Calvetti, N. Cartiglia, A. Ceccucci, P. Cenci, C. Cerri, C. Cheshkov, J.B. Chèze, M. Clemencic, G. Collazuol, F. Costantini, A. Cotta Ramusino, D. Coward, D. Cundy, A. Dabrowski, P. Dalpiaz, C. Damiani, M. De Beer, J. Derré, H. Dibon, L. DiLella, N. Doble, K. Eppard, V. Falaleev, R. Fantechi, M. Fidecaro, L. Fiorini, M. Fiorini, T. Fonseca Martin, P.L. Frabetti, L. Gatignon, E. Gersabeck, A. Gianoli, S. Giudici, A. Gonidec, E. Goudzovski, S. Goy Lopez, M. Holder, P. Hristov, E. Iacopini, E. Imbergamo, M. Jeitler, G. Kalmus, V. Kekelidze, K. Kleinknecht, V. Kozhuharov, W. Kubischta, G. Lamanna, C. Lazzeroni, M. Lenti, L. Litov, D. Madigozhin, A. Maier, I. Mannelli, F. Marchetto, G. Marel, M. Markytan, P. Marouelli, M. Martini, L. Masetti, E. Mazzucato, A. Michetti, I. Mikulec, M. Misheva, N. Molokanova, E. Monnier, U. Moosbrugger, C. Morales Morales, D.J. Munday, A. Nappi, G. Neuhofer, A. Norton, M. Patel, M. Pepe, A. Peters, F. Petrucci, M.C. Petrucci, B. Peyaud, M. Piccini, G. Pierazzini, I. Polenkevich, Yu. Potrebenikov, M. Raggi, B. Renk, P. Rubin, G. Ruggiero, M. Savrié, M. Scarpa, M. Shieh, S. Shkarovskiy, M.W. Slater, M. Sozzi, S. Stoynev, E. Swallow, M. Szleper, M. Valdata-Nappi, B. Vallage, M. Velasco, M. Veltri, S. Venditti, M. Wache, H. Wahl, A. Walker, R. Wanke, L. Widhalm, A. Winhart, R. Winston, M.D. Wood, S.A. Wotton, A. Zinchenko, M. Ziolkowski. 


\section{Introduction}

The chiral perturbation theory (ChPT), is a widely used effective field theory describing lowenergy quantum chromodynamics (QCD), valid below a scale $O(1 \mathrm{GeV})$. Radiative kaon decays are of particular interest in testing this theory, allowing to measure a subset of the ChPT chiral Lagrangian free parameters [1]. The NA48 and NA48/2 experiments already widely contributed in measuring these parameters. The first observation of the $K^{ \pm} \rightarrow \pi^{ \pm} \pi^{0} e^{+} e^{-}$decays completes this effort.

Since the late ' 60 Kaon decays have played a major role in establishing the quark mixing flavour structure of the Standard Model[2]. Even recently, tension were observed in tests of the unitarity of the CKM matrix, which suggest a better precision is needed in the measurement of the semi-leptonic Kaon decays form factors. In this paper we will report on the most recent measurement of the Ke3 and $\mathrm{K} \mu 3$ form factors obtained by the NA48/2 collaboration.

\section{The NA48/2 experiment}

The NA48/2 experiment at the CERN SPS was designed to measure the charge asymmetry in the $K^{ \pm} \rightarrow 3 \pi$ decay modes [3]. During the 2003-2004 data taking period large samples of charged kaon decays were collected using simultaneous narrow momentum band $K^{+}$and $K^{-}$beams, $60 \mathrm{GeV} / c \pm$ $3.8 \%$ (rms), originating from primary $400 \mathrm{GeV} / c$ protons impinging on a beryllium target.

The momenta of charged particles originating from $K^{ \pm}$decays were measured in a magnetic spectrometer. The spectrometer was composed of four drift chambers (DCH), housed in a tank filled with helium at nearly atmospheric pressure, two on each side of a dipole magnet providing a momentum kick in the horizontal plane of $\Delta p=120 \mathrm{MeV} / c$. The achieved momentum resolution was $\sigma_{p} / p=(1.02 \oplus 0.044 \cdot p) \%(p$ in $\mathrm{GeV} / c)$.

A liquid krypton electromagnetic calorimeter ( $\mathrm{LKr}$ ), an almost homogeneous ionization chamber with an active volume of $7 \mathrm{~m}^{3}$, was placed further downstream, The energies of photons and electrons were measured with resolutions $\sigma_{E} / E=(3.2 / \sqrt{E} \oplus 9.0 / E \oplus 0.42) \%$. The transverse positions of isolated showers were measured with a spatial resolution $\sigma_{x}=\sigma_{y}=(0.42 / \sqrt{E} \oplus 0.06)$ $\mathrm{cm}$, and the shower time resolution was $2.5 \mathrm{~ns} / \sqrt{E}(E \mathrm{in} \mathrm{GeV})$. An iron/scintillator hadronic calorimeter and muon detectors were located further downstream.

More details about the detector, the beam line, and trigger system can be found in [3]. A detailed description of the detector can be found in [4].

\section{Introduction to $K^{ \pm} \rightarrow \pi^{ \pm} \pi^{0} e^{+} e^{-}$theoretical framework}

The never observed radiative decay $K^{ \pm} \rightarrow \pi^{ \pm} \pi^{0} e^{+} e^{-}$, proceeds through virtual photon exchange followed by internal conversion into an electron-positron pair, i.e. $\quad K^{ \pm} \rightarrow \pi^{ \pm} \pi^{0} \gamma^{*} \rightarrow$ $\pi^{ \pm} \pi^{0} e^{+} e^{-}$.

Two different mechanisms can produce the virtual $\gamma^{*}$ : Inner Bremsstrahlung (IB) where the $\gamma^{*}$ is emitted by initial or final state radiation of one of the charged mesons, and Direct Emission (DE) where the $\gamma^{*}$ is radiated off at the weak vertex. For this reason, the differential decay rate consists of three contributions: the long-distance dominated IB contribution, the electric $\mathrm{E}$ and magnetic $\mathrm{M}$ 
parts of the DE component, and their interference, which includes the different contributions, IB-E, IB-M and E-M.

After an early paper in 2001[5], only recently few theoretical publications related to the $K^{ \pm} \rightarrow \pi^{ \pm} \pi^{0} e^{+} e^{-}$mode $[6,7]$ were produced, but still no experimental observation exist. On the basis of the NA48/2 measurement of the magnetic and electric terms involved in the $K^{ \pm} \rightarrow \pi^{ \pm} \pi^{0} \gamma$ decay [8], the authors of [6] were able to predict, the branching ratios of IB, DE and INT components of the $K^{ \pm} \rightarrow \pi^{ \pm} \pi^{0} e^{+} e^{-}$decay. Recently a revised work [9], in which the interference term is reevaluated using more realistic experimental inputs and fewer theoretical assumptions, was posted.

Authors of [6] explicitly define the different contributions to the squared amplitude of the decay

$$
\sum_{\text {spins }}|M|^{2}=\frac{2 e^{2}}{q^{4}}\left[\sum_{i=1}^{3}\left|F_{i}\right|^{2} T_{i i}+2 R e \sum_{i<j}^{3}\left(F_{i}^{*} F_{j}\right) T_{i j}\right],
$$

where $F_{i}$ and $T_{i j}$ are complex form factors and kinematic expressions defined in [6]. The complex form factors $F_{1}^{D E}, F_{2}^{D E}$ correspond to the electric part of DE and make use of the ChPT counterterms $N_{E}^{(0,1,2)}$ while $F_{3}^{D E}$ corresponds to the magnetic part of DE and makes use of the counterterm $N_{M}^{(0)}$. Numerical values of the counterterms were estimated [9] using experimental measurements of form factors in the related modes $K^{ \pm} \rightarrow \pi^{ \pm} \gamma^{*}, K_{S} \rightarrow \pi^{0} \gamma^{*}$ and $K^{ \pm} \rightarrow \pi^{ \pm} \pi^{0} \gamma$.

\section{First observation of $K^{ \pm} \rightarrow \pi^{ \pm} \pi^{0} e^{+} e^{-}$}

The whole data sample recorded by the NA48/2 experiment in $2003-04,1.7 \times 10^{11}$ kaon decays, has been scanned, searching for the unobserved $K^{ \pm} \rightarrow \pi^{ \pm} \pi^{0} e^{+} e^{-}$decay.
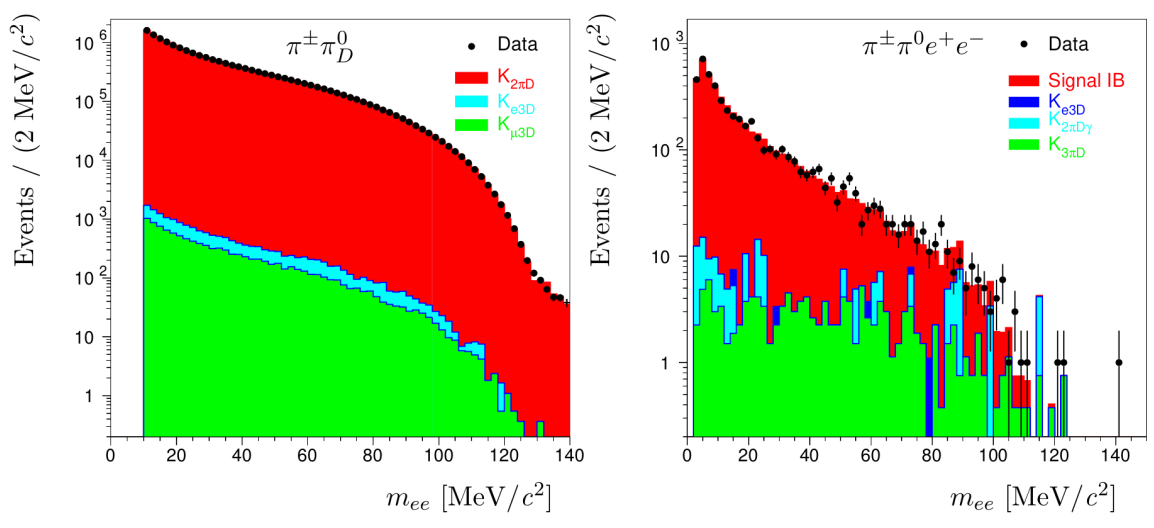

Figure 1: Reconstructed $e^{+} e^{-}$mass distribution for the normalization (left) and signal (right) candidates with the lower cuts of 10 and $3 \mathrm{MeV} / \mathrm{c}^{2}$, respectively. Simulated background and normalization (signal) contributions are also displayed[10].

A sample of 4919 decay candidates, with an estimated background contribution of $4.9 \%$ dominated by $\pi^{ \pm} \pi^{0} \pi_{D}^{0}$ with a missing $\gamma$, has been selected, providing the first observation of this decay mode. Details on the selection algorithm used in the data analysis can be found in [10]. The branching ratio has been measured relative to the large data sample available for the $K^{ \pm} \rightarrow \pi^{ \pm} \pi^{0}$ 
mode followed by a Dalitz decay $\pi_{D}^{0} \rightarrow e^{+} e^{-} \gamma$, collected with the same L0 and L1 trigger selection process, allowing to minimize trigger related systematic effects. Fig. 1 shows the reconstructed $e^{+} e^{-}$ mass distribution for the normalization (left) and signal (right) candidates[10]. Both background and normalization (signal) simulations, scaled to the number of observed candidates, show a good agreement with the data distributions.

The total $K^{ \pm} \rightarrow \pi^{ \pm} \pi^{0} e^{+} e^{-}$BR has been found to be[10]:

$$
B R\left(K^{ \pm} \rightarrow \pi^{ \pm} \pi^{0} e^{+} e^{-}\right)=\left(4.237 \pm 0.063_{\text {stat }} \pm 0.033_{\text {syst }} \pm 0.126_{\text {ext }}\right) \times 10^{-6},
$$

in agreement with ChPT predictions for the total $B R\left(K^{ \pm} \rightarrow \pi^{ \pm} \pi^{0} e^{+} e^{-}\right)=4.229 \times 10^{-6}$, including all DE and INT terms. It should be however noted, that the prediction above does not includes any radiative or isospin breaking corrections.

Despite the limited statistics available, a study of the kinematic of the decay has been performed to extract information on the magnetic (M) and interference (IB-E) contributions with respect to the dominant inner bremsstrahlung (IB). The relative contribution, $(\mathrm{M}) / \mathrm{IB}=\left(1.14 \pm 0.43_{\text {stat }}\right) \times 10^{-2}$, is found consistent with the theoretical expectation of $\left(1.41 \pm 0.14_{\text {ext }}\right) \times 10^{-2}$. The relative IB-E contribution, (IB-E)/IB $=\left(-0.14 \pm 0.36_{\text {stat }}\right) \times 10^{-2}$, is also in agreement with the prediction of $\left(-0.39 \pm 0.28_{\text {ext }}\right) \times 10^{-2}$ but with limited significance due to the lack of data statistics in the high $m_{e e}$ region.

Several CP-violating asymmetries and a long-distance P-violating asymmetry have been identified in [6]. All the asymmetries have been evaluated in the NA48/2 analysis and found to be consistent with zero, allowing to set the following upper limits at $90 \% \mathrm{CL}$ :

$$
\text { - }\left|A_{C P}\right|<4.8 \times 10^{-2}, \quad\left|A_{C P}^{\phi^{*}}\right|<3.1 \times 10^{-2}, \quad\left|A_{C P}^{\tilde{\phi}}\right|<2.5 \times 10^{-2}, \quad\left|A_{P}^{(L)}\right|<2.1 \times 10^{-2}
$$

All of the upper limits are limited by the statistics of the available data sample.

\section{Semileptonic kaon decays form factor measurements}

Within the Standard Model (SM), semileptonic kaon decays can provide the most experimentally accurate and theoretically cleanest determination of the magnitude of the element $V_{u s}$ of the Cabibbo-Kobayashi-Maskawa (CKM) matrix[11]. In order to precisely measure the FF avoiding bias coming from the online trigger selection, a dedicated data set was recorded by NA48/2 at reduced beam intensity using a minimum bias trigger during a 52-hour long data-taking period in 2004.

The starting point to extract the value of $\left|V_{u s}\right|$ from semileptonic decays is the photon inclusive rate of $K \rightarrow \pi \ell v(\gamma)(\ell=\mathrm{e}, \mu)$

$$
\Gamma\left(K_{\ell 3(\gamma)}\right)=\frac{C_{K}^{2} G_{F}^{2} m_{K}^{5}}{192 \pi^{3}} S_{E W}\left|V_{u s}\right|^{2}\left|f_{+}(0)\right|^{2} I_{K}^{\ell}\left(\lambda_{+0}\right)\left(1+\delta_{S U(2)}+\delta_{E M}^{\ell}\right)^{2}
$$

where the notation in [11] has been used. To extract $\left|V_{u s}\right|$ from $K \ell 3$ decays using Eq. 3, we need to measure one or more $K \ell 3$ decay rates and make use of theoretical inputs for $f_{+}(0), \delta_{S U(2)}, \delta_{E M}^{\ell}$. 


\section{1 $K \ell 3$ form factor parameterisations}

The hadronic matrix element of $K \ell 3$ decays is described by two dimensionless form factors $f_{ \pm}(t)$, which depend on the squared four-momentum $t=\left(p_{K}-p_{\pi}\right)^{2}$ transferred to the leptonneutrino system. Usually, form factors are re-formulated to express the vector $f_{+}(t)$ and scalar $f_{0}(t)$ exchange contributions, and $f_{0}(t)$ can be described as a linear combination of $f_{ \pm}(t)$.

\begin{tabular}{|c|c|c|}
\hline & $f_{+}(t)$ & $f_{0}(t)$ \\
\hline Taylor expansion & $1+\lambda_{+}^{\prime} \frac{t}{m_{\pi^{+}}^{2}}+\frac{1}{2} \lambda_{+}^{\prime \prime}\left(\frac{t}{m_{\pi^{+}}^{2}}\right)^{2}$ & $1+\lambda_{0} \frac{t}{m_{\pi^{+}}^{2}}$ \\
\hline Pole & $\frac{M_{V}^{2}}{M_{V}^{2}-t}$ & $\frac{M_{S}^{2}}{M_{S}^{2}-t}$ \\
\hline Dispersive & $\exp \left(\frac{\Lambda_{+}+H(t)}{m_{\pi^{+}}^{2}} t\right)$ & $\exp \left(\frac{\ln C-G(t)}{m_{K}^{2}-m_{\pi^{0}}^{2}} t\right)$ \\
\hline
\end{tabular}

Table 1: Form factor parameterizations used in NA48/2 analysis.

\subsection{NA48/2 $K \ell 3$ form factor result}

A new measurements of the FF has been obtained by NA48/2 using a total of $4.4(2.3) \times 10^{6}$ reconstructed $K e 3(K \mu 3)$ candidates[12]. The fit results are listed in Tables 2, 3 and 4 for $K_{e 3}^{ \pm}, K_{\mu 3}^{ \pm}$ and the combined analysis, respectively. The fit quality is always satisfactory, as indicated by the $\chi^{2}$ values. The form factor measurements, obtained from $K_{e 3}^{ \pm}$and $K_{\mu 3}^{ \pm}$decays data set, are in good agreement. The correlation includes both statistical and systematic uncertainties.

\begin{tabular}{|l|rr|c|c|}
\hline & $\lambda_{+}^{\prime}$ & $\lambda_{+}^{\prime \prime}$ & $M_{V}\left(\mathrm{MeV} / c^{2}\right)$ & $\Lambda_{+}$ \\
\hline Central values & 24.26 & 1.64 & 885.2 & 24.94 \\
\hline Statistical error & 0.78 & 0.30 & 3.3 & 0.21 \\
\hline Systematic error & 1.30 & 0.39 & 7.2 & 0.64 \\
\hline Total error & 1.51 & 0.49 & 7.9 & 0.67 \\
\hline Correlation coefficient & -0.929 & - & - \\
\hline$\chi^{2} / \mathrm{NDF}$ & $569.1 / 687$ & $568.9 / 688$ & $569.0 / 688$ \\
\hline
\end{tabular}

Table 2: Form factor results of the $K_{e 3}^{ \pm}$analysis[12]. The units of $\lambda_{+}^{\prime}, \lambda_{+}^{\prime \prime}$ and $\Lambda_{+}$values and errors are $10^{-3}$.

\begin{tabular}{|l|rrr|rr|rr|}
\hline & $\lambda_{+}^{\prime}$ & $\lambda_{+}^{\prime \prime}$ & $\lambda_{0}$ & $m_{V}\left(\mathrm{MeV} / c^{2}\right)$ & $m_{S}\left(\mathrm{MeV} / c^{2}\right)$ & $\Lambda_{+}$ & $\ln C$ \\
\hline Central values & 24.27 & 1.83 & 14.20 & 878.4 & 1214.8 & 25.36 & 182.17 \\
\hline Statistical error & 2.88 & 1.05 & 1.14 & 8.8 & 23.5 & 0.58 & 6.31 \\
\hline Systematic error & 2.89 & 1.09 & 1.07 & 8.3 & 49.2 & 0.72 & 14.45 \\
\hline Total error & 4.08 & 1.52 & 1.57 & 12.1 & 54.5 & 0.92 & 15.76 \\
\hline Correlation coefficients & $-0.974\left(\lambda_{+}^{\prime} / \lambda_{+}^{\prime \prime}\right)$ & 0.029 & & 0.104 \\
& $0.511\left(\lambda_{+}^{\prime} / \lambda_{0}\right)$ & & & & \\
& $-0.513\left(\lambda_{+}^{\prime \prime} / \lambda_{0}\right)$ & & & & & \\
\hline$\chi^{2} / \mathrm{NDF}$ & $409.9 / 381$ & $409.9 / 382$ & & $410.3 / 382$ \\
\hline
\end{tabular}

Table 3: Form factor results of the $K_{\mu 3}^{ \pm}$analysis[12]. The units of $\lambda_{+}^{\prime}, \lambda_{+}^{\prime \prime}, \lambda_{0}, \Lambda_{+}$and $\ln C$ values and errors are $10^{-3}$.

An update on the $\left|V_{u s}\right|$ extraction including the new FF measurements by NA48/2 can be found in [13]; 


\begin{tabular}{|l|rrr|rr|rr|}
\hline & $\lambda_{+}^{\prime}$ & $\lambda_{+}^{\prime \prime}$ & $\lambda_{0}$ & $m_{V}\left(\mathrm{MeV} / c^{2}\right)$ & $m_{S}\left(\mathrm{MeV} / c^{2}\right)$ & $\Lambda_{+}$ & $\ln C$ \\
\hline Central values & 24.24 & 1.67 & 14.47 & 884.4 & 1208.3 & 24.99 & 183.65 \\
\hline Statistical error & 0.75 & 0.29 & 0.63 & 3.1 & 21.2 & 0.20 & 5.92 \\
\hline Systematic error & 1.30 & 0.41 & 1.17 & 6.7 & 47.5 & 0.62 & 14.25 \\
\hline Total error & 1.50 & 0.50 & 1.32 & 7.4 & 52.1 & 0.65 & 15.43 \\
\hline Correlation coefficient & $-0.934\left(\lambda_{+}^{\prime} / \lambda_{+}^{\prime \prime}\right)$ & 0.374 & & & 0.354 \\
& $0.118\left(\lambda_{+}^{\prime} / \lambda_{0}\right)$ & & & & \\
\hline$\chi^{2} / \mathrm{NDF}$ & $0.091\left(\lambda_{+}^{\prime \prime} / \lambda_{0}\right)$ & & & & & \\
& $979.6 / 1070$ & & $979.3 / 1071$ & & & \\
\hline
\end{tabular}

Table 4: Form factor results of the joint $K_{\ell 3}^{ \pm}$analysis[12]. The units of $\lambda_{+}^{\prime}, \lambda_{+}^{\prime \prime}, \lambda_{0}, \Lambda_{+}$and $\ln C$ values and errors are $10^{-3}$.

\section{Conclusions}

In this paper we reported the most recent results obtained by the NA48/2 experiment on rare kaon decays. The first observation of the $K^{ \pm} \rightarrow \pi^{ \pm} \pi^{0} e^{+} e^{-}$allowed the determination of the branching ratio in the full kinematic region, and the investigation of DE and INT components. A new measurement of the FF for the $K_{\ell 3}$ decays has been reported, obtaining precise determination of the FF parameter for most common parameterisation, using both separated and combined $\mathrm{Ke} 3$ and $K \mu 3$ decays data samples.

\section{References}

[1] G. D’Ambrosio, PoS EFT09, 061 (2009)

[2] V. Cirigliano, G. Ecker, H. Neufeld, A. Pich and J. Portolés, Rev. Mod. Phys. 84 (2012) 399.

[3] J.R. Batley et al. [NA48/2 Collaboration], Eur. Phys. J. C 52 (2007) 875.

[4] V. Fanti et al. [NA48 Collaboration], Nucl. Instrum. Methods A 574 (2007) 443.

[5] H. Pichl, Eur. Phys. J. C 20 (2001) 371.

[6] L. Cappiello, O. Catà, G. D’Ambrosio and D.N. Gao, Eur. Phys. J. C 72 (2012) 1872.

[7] S.R. Gevorkyan and M.H. Misheva, Eur. Phys. J. C 74 (2014) 2860.

[8] J.R. Batley et al. [NA48/2 Collaboration], Eur. Phys. J. C 68 (2010) 75.

[9] L. Cappiello, O. Catà and G. D’Ambrosio, Eur. Phys. J. C 78 (2018) 265.

[10] J. R. Batley et al. [NA48/2], Phys. Lett. B 788, 552-561 (2019)

[11] M. Antonelli et al. (FlaviaNet Working Group on K Decays), Eur. Phys. J. C 69 (2010) 399.

[12] J. R. Batley et al. [NA48/2], JHEP 10, 150 (2018)

[13] "The status of Vus" M. Moulson Workshop on Top-Row CKM Unitarity Texas A\&M University Jan 2019 